\title{
Targeted Delivery of Therapeutic Agents by Smart Nanocarrier for Treatment of Parkinson's Disease: A Novel Brain Targeting Approach
}

\author{
Fatemeh Moradi ${ }^{1}$, Houman Parsaie ${ }^{1,2}$ and Enam Alhagh Charkhat Gorgich (iD ${ }^{1,2,3,{ }^{*}}$ \\ ${ }^{1}$ Department of Anatomical Sciences, School of Medicine, Iran University of Medical Sciences, Tehran, Iran \\ ${ }^{2}$ Students' Scientific Association of Anatomical Sciences, School of Medicine, Iran University of Medical Sciences, Tehran, Iran \\ ${ }^{3}$ Department of Histology, School of Medicine, Zahedan University of Medical Sciences, Zahedan, Iran \\ "Corresponding author: Department of Histology, School of Medicine, Zahedan University of Medical Sciences, Zahedan, Iran. Email: enam.gorgich@yahoo.com
}

Received 2019 March 03; Revised 2019 March 07; Accepted 2019 March 09.

Keywords: Parkinson's Disease, Nanomedicine, Liposomes, Drug Delivery Systems, Targeted Therapy

\section{Dear Editor,}

Parkinson's disease (PD) stays a standout amongst the most well-known and partially treatable age-related neurodegenerative movement disorders. It is described by irreversible demolition of dopamine-producing neurons in the substantia nigra and formation of $\alpha$-synucleincontaining Lewy bodies (1). The principal motor manifestations such as resting tremor, bradykinesia, rigidity and postural imbalance occur based on the reduction in synthesis of dopamine (DA) in the striatum caused by the progressive degeneration of DAergic neurons, subsequently, volumetric changes occur in different regions of the brain of the patients $(1,2)$.

Currently, the foundation of PD treatment is based on the DA substitution scenario utilizing the precursor, LDOPA. The strategy is effective only for PD patients in early stages of the disease, while just $1 \%$ given L-DOPA could reach the brain and continued treatment with L-DOPA often causes the unwanted secondary effects owing to its non-targeting. On the other hand, L-DOPA decarboxylase, the enzyme that plays an essential role in converting LDOPA to DA, is less active in PD patients (3).

$\mathrm{N}-3,4$-bis(pivaloyloxy)-dopamine (BPD) is a brainspecific derivative of DA and consists of two dipivaloyloxy groups for lipophilic modification and it is more stable than DA; in comparison with L-DOPA, BPD displayed improved brain targeting capability and boost curative properties (4). Therefore, BPD demonstrates a promising therapeutic candidate for PD treatment. Nevertheless, BPD has a short half-life in plasma and a certain portion of BPD go through the brain physiological barriers passively (4, 5).

Nowadays, to overcome obstacles and improve brain- targeted delivery of L-DOPA, nano-based drug delivery systems have been developed. Liposomes are sphericalshaped nanocarriers that are composed of one or more phospholipid bilayers (6). The most important advantages of liposomes for drug delivery include: the ability of encapsulation of hydrophilic and lipophilic therapeutic agents, the ability to transit the blood-brain barrier(BBB) as a main challenge in the treatment of central nervous system diseases, the surface modifications through conjugation with a variety of targeted ligands, including transferrin, insulin, low-density lipoprotein receptors (7) in order to improve the brain targeting, being non-toxic and possessing FDA approval $(6,8)$.

Rabies virus glycoprotein (RVG-29) is a brain-targeting peptide with 29 amino-acid that can intracerebrally deliver therapeutic agents (9). RVG-29 distinctively binds to the acetylcholine receptors (AchR) on DAergic cells and endothelial cells of the brain capillaries (10). This braintargeting peptide has been proven effective in facilitating the transportation of exosomes, genes, mirRNAs, siRNAs, and DNA over the BBB through AchR-mediated endocytosis (11). Furthermore, RVG-29 is theorized to attain a targeted delivery system to DAergic cells through the interplay among RVG-29 and AchR (5).

As per the above-mentioned, it can be expected that due to the characteristics of RVG-liposomes loaded by BPD, functionalized liposomes should facilitate crossing from the BBB and improve brain distribution of BPD (5). Therefore, nanoliposome-based targeted drug delivery system design can be a promising strategy for the targeted treatment of PD and even other CNS disorders $(5,12)$.

Finally, since RVG-liposomes loaded by BPD were potentially effective for brain-targeted drug delivery and be- 
cause of encapsulation of BPD which produces a therapeutic effect, this drug delivery system could be the subject of further studies in order to assess its distributions in offtarget tissues and further ensure the biosafety of this delivery system. This strategy could be the subject of further research at molecular and cellular levels for final approval and clinical application.

In conclusion, based on the results of recent studies (46) it appears that RVG-29-functionalized drug delivery systems could be beneficial for the targeted therapy of PD and can be considered as a novel promising fashion to targeted drug delivery to the other CNS disorders.

\section{Footnotes}

Conflict of Interests: The authors have no conflict of interests to declare.

Funding/Support: The authors of this paper appreciate the Zahedan University of Medical Sciences and the Iran University of Medical Sciences that assisted in the development of the projects related to this issue.

\section{References}

1. Tolosa E, Wenning G, Poewe W. The diagnosis of parkinson's disease. Lancet Neurol. 2006;5(1):75-86. doi: 10.1016/S1474-4422(05)70285-4.

2. Heidari Z, Moghtaderi A, Mahmoudzadeh-Sagheb H, Gorgich EA. Stereological evaluation of the brains in patients with parkinson's disease compared to controls. Rev Romana Med Lab. 2017;25(3):265-74. doi: 10.1515/rrlm-2017-0010.

3. Huot P, Johnston TH, Koprich JB, Fox SH, Brotchie JM. The pharmacology of L-DOPA-induced dyskinesia in Parkinson's disease. Pharmacol Rev. 2013;65(1):171-222. doi: 10.1124/pr.111.005678. [PubMed: 23319549].
4. Li Y, Zhou Y, Qi B, Gong T, Sun X, Fu Y, et al. Brain-specific delivery of dopamine mediated by n,n-dimethyl amino group for the treatment of Parkinson's disease. Mol Pharm. 2014;11(9):3174-85. doi: 10.1021/mp500352p. [PubMed: 25072272].

5. Qu M, Lin Q, He S, Wang L, Fu Y, Zhang Z, et al. A brain targeting functionalized liposomes of the dopamine derivative $\mathrm{N}$ 3,4-bis(pivaloyloxy)-dopamine for treatment of Parkinson's disease. J Control Release. 2018;277:173-82. doi: 10.1016/j.jconrel.2018.03.019. [PubMed: 29588159].

6. Belfiore L, Saunders DN, Ranson M, Thurecht KJ, Storm G, Vine KL. Towards clinical translation of ligand-functionalized liposomes in targeted cancer therapy: Challenges and opportunities. JControl Release. 2018;277:1-13. doi:10.1016/j.jconrel.2018.02.040. [PubMed: 29501721].

7. Vlieghe $\mathrm{P}$, Khrestchatisky M. Medicinal chemistry based approaches and nanotechnology-based systems to improve CNS drug targeting and delivery. Med Res Rev. 2013;33(3):457-516. doi: 10.1002/med.21252. [PubMed: 22434495].

8. Sanchez-Moreno P, Ortega-Vinuesa JL, Peula-Garcia JM, Marchal JA, Boulaiz H. Smart drug-delivery systems for cancer nanotherapy. Curr Drug Targets. 2018;19(4):339-59. doi: 10.2174/1389450117666160527142544. [PubMed: 27231107].

9. Liu Y, Li J, Shao K, Huang R, Ye L, Lou J, et al. A leptin derived 30amino-acid peptide modified pegylated poly-L-lysine dendrigraft for brain targeted gene delivery. Biomaterials. 2010;31(19):5246-57. doi: 10.1016/j.biomaterials.2010.03.011. [PubMed: 20382424].

10. Kumar P, Wu H, McBride JL, Jung KE, Kim MH, Davidson BL, et al. Transvascular delivery of small interfering RNA to the central nervous system. Nature. 2007;448(7149):39-43. doi: 10.1038/nature05901. [PubMed: 17572664].

11. Liu Y, Li D, Liu Z, Zhou Y, Chu D, Li X, et al. Targeted exosome-mediated delivery of opioid receptor $\mathrm{Mu}$ siRNA for the treatment of morphine relapse. Sci Rep. 2015;5:17543. doi: 10.1038/srep17543. [PubMed: 26633001]. [PubMed Central: PMC4668387].

12. Lam FC, Morton SW, Wyckoff J, Vu Han TL, Hwang MK, Maffa A, et al. Enhanced efficacy of combined temozolomide and bromodomain inhibitor therapy for gliomas using targeted nanoparticles. Nat Commun. 2018;9(1):1991. doi:10.1038/s41467-018-04315-4. [PubMed: 29777137]. [PubMed Central: PMC5959860]. 